
\title{
Tuneable topological domain wall states in engineered atomic
} chains

\author{
Md Nurul Huda ${ }^{1}$, Shawulienu Kezilebieke $\mathbb{D}^{1}$, Teemu Ojanen ${ }^{2,3}$, Robert Drost ${ }^{4}$ and Peter Liljeroth $\mathbb{D}^{1 凶}$
}

Topological modes in one- and two-dimensional systems have been proposed for numerous applications utilizing their exotic electronic responses. The 1D, zero-energy, topologically protected end modes can be realized in structures implementing the Su-Schrieffer-Heeger (SSH) model. While the edge modes in the SSH model are at exactly the mid-gap energy, other paradigmatic $1 \mathrm{D}$ models such as trimer and coupled dimer chains have non-zero energy boundary states. However, these structures have not been realized in an atomically tuneable system that would allow explicit control of the edge modes. Here, we demonstrate atomically controlled trimer and coupled dimer chains realized using chlorine vacancies in the $\mathrm{c}(2 \times 2)$ adsorption layer on $\mathrm{Cu}(100)$. This system allows wide tuneability of the domain wall modes that we experimentally demonstrate using low-temperature scanning tunneling microscopy (STM).

npj Quantum Materials (2020)5:17; https://doi.org/10.1038/s41535-020-0219-3

\section{INTRODUCTION}

The essential physics of quantum materials can be often captured using tight-binding (TB) models describing hopping between localized electronic orbitals. The argument can also be reversed: given sufficient control, it is possible to realize experimentally artificial materials by a suitable arrangement of coupled "sites". This can be achieved using atom manipulation by the tip of a scanning tunneling microscope (STM), which allows placing each atom individually into a well-defined, predetermined position ${ }^{1-10}$. It is thus possible to build designer quantum materials with ultimate control over their electronic structure through atomic assemblies. This has been used to demonstrate the formation of quantum confined one- or two-dimensional electronic systems, and artificial lattices with e.g. honeycomb and Lieb symmetries $2,4,6,8-15$

Topological modes in one- and two-dimensional systems have been proposed for numerous applications utilizing their exotic electronic responses ${ }^{16-24}$. Atomic manipulation can naturally also be applied to topological materials and systems with interface and edge states can be constructed ${ }^{8}$. In one-dimensional (1D) systems, the dimer chain (Fig. 1a, Su-Schrieffer-Heeger model realized in e.g. polyacetylene) is the prototypical example of a 1D topological material ${ }^{25}$. The system comprises of dimers with a strong hopping between the sites $\left(t_{1}\right)$, and weaker hopping between the dimers $\left(t_{2}\right)$. Depending on the choice of the unit cell (see Fig. 1a), the chain can exist in two, topologically distinct, ground states. The bulk band structure is unchanged when comparing two infinite chains displaced by a fraction of a unit cell. However, merging two such displaced gapped chains gives rise to a domain wall and an in-gap state spatially localized in the vicinity of the domain wall $^{8,25-27}$. These zero-energy modes have been experimentally implemented in atomic-scale solid-state structures and in ultracold atomic gases ${ }^{8,28}$, and analogous systems have been realized in graphene nanoribbons $s^{23,29,30}$. This state is protected in the sense that it is impossible to get rid of it without closing the bulk energy gap and it will always occur when joining two dimer chains with displaced unit cells. At half-filling, the existence of this state can be understood in the form of a soliton state with a polarization charge $\pm e / 2$ on the boundary of the two ground states of dimer chain. For this simplest realization of a dimer chain, the in-gap state is exactly at the mid-gap energy.

The existence of a domain wall state at exactly mid-gap energy does not necessarily occur for 1D chains consisting of sites with different on-site energies or more complicated unit cells with three or more atoms ${ }^{21,22,31-35}$. While the states still necessarily occur at the domain wall, their energies within the gap can be tuned. A seminal contribution on self-assembled indium atomic wires on $\mathrm{Si}(111)$ demonstrated the formation of coupled dimer chains with four topological distinct bulk phases ${ }^{21}$. Cheon et al. identified different kinds of interfaces between the phases and showed that the interface states in these double Peierls chains can be understood as topological chiral solitons ${ }^{21,22}$.

The self-assembled chains can be influenced by defects and other imperfections, and they have a stochastic distribution of domain walls with different structures and properties ${ }^{21,36-39}$. This can be overcome using atomic manipulation that allows the realization of perfect, defect-free structures with predetermined types of domain walls. We use atomic manipulation with a lowtemperature STM to realize paradigmatic 1D model systems of trimer and coupled dimer chains with precisely controlled domain wall structures. Here, we use the localized electronic states hosted in chlorine vacancies in the chlorine $\mathrm{c}(2 \times 2)$ structure on $\mathrm{Cu}(100)$ as building blocks, which have recently be used to create artificial systems with designer electronic structures ${ }^{7-9}$. We focus on linear systems where the unit cell is more complicated (trimer and coupled dimer chains) and demonstrate the formation of tuneable interface states between different ground states of the system. The domain walls between different trimer and coupled dimer states can in principle be used to prepare localized and welldefined fractional charges and to manipulate them. In the future, these modes may find applications in exotic quantum devices with atomically well-defined geometries ${ }^{25,31,40,41}$.

\footnotetext{
${ }^{1}$ Department of Applied Physics, Aalto University School of Science, PO Box 15100, 00076 Aalto, Finland. ${ }^{2}$ Computational Physics Laboratory, Physics Unit, Faculty of Engineering and Natural Sciences, Tampere University, PO Box 692, Fl-33014 Tampere, Finland. ${ }^{3}$ Helsinki Institute of Physics, PO Box 64 , Fl-00014 Helsinki, Finland. ${ }^{4}$ Nanoscale Science Department, Max-Planck-Institute for Solid State Research, Heisenbergstrasse 1, D-70569 Stuttgart, Germany. ${ }^{\bowtie}$ email: peter.liljeroth@aalto.fi
} 


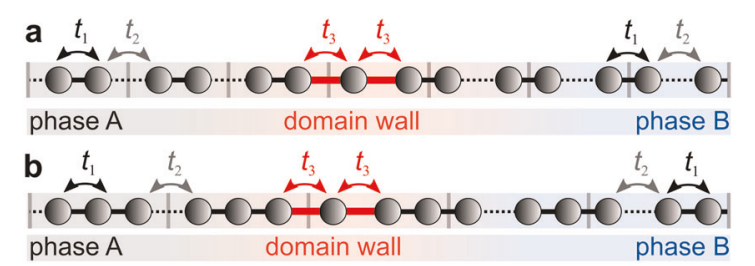

C
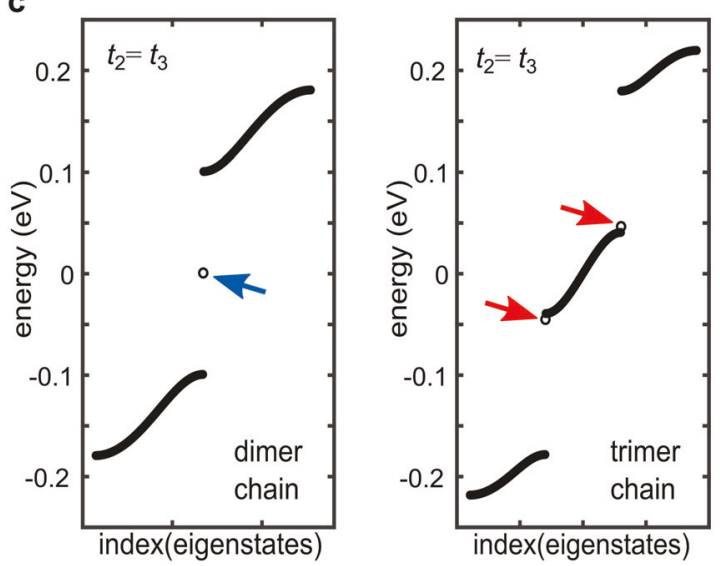

Fig. 1 Domain walls in dimer and trimer chains. a, b Schematic of dimer (a) and trimer (b) chain with a domain wall. c Calculated energy spectra of finite dimer and trimer chains hosting a domain wall with $t_{1}=0.14 \mathrm{eV}, t_{2}=0.04 \mathrm{eV}^{8}$ calculated with 300 unit cells on both sides of the domain wall. While the in-gap state in a dimer chain is pinned to mid-gap, domain wall in a trimer chain results tunable in-gap states depending on the hopping $t_{3}$.

\section{RESULTS AND DISCUSSION}

Figure $1 \mathrm{~b}$ shows a schematic of a trimer chain. Similarly to dimer chains, joining sections of trimer chains with different unit cells necessarily results in the formation of a domain wall that cannot be removed from the system by a local perturbation. Perfectly trimerized chains (intratrimer hopping $t_{1}$, and intertrimer hopping $t_{2}$ ) have three distinct topological phases and an electronic structure with three separate bands. In a system containing a domain wall, localized states appear in the band gaps ${ }^{31,42}$. Domain walls host a fractional charge of $\pm 1 / 3 e$ or $\pm 2 / 3 e$ depending on domain wall type and the chemical potential of the system. These charges appear due to the mismatch of the unit cells on the opposite sides of the domain wall and cannot be removed without changing the bulk structure of the chain ${ }^{31}$. However, the energy position of the state associated with domain wall is not fixed and can be moved within the gap; the states are movable, but irremovable. The electronic structure based on TB calculations in finite dimer and trimer chains are illustrated in Fig. 1c. The TB parameters correspond to the experimental values of the chlorine vacancy system ${ }^{8}$ (details of the TB calculations are given in Supplementary Note 1). Analyzing the nature and the number of the localized states, it is clear that they arise from bonding and antibonding combinations between the states on the domain wall site and the nearest trimer units.

Figure $2 \mathrm{a}$ shows a realization of a trimer chain with a domain wall in the chlorine vacancy system introduced in refs. ${ }^{7-9}$. Sample preparation and the details of the STM experiments are described in the Methods section. In this experimental system, it is difficult to access the higher energy gap of the trimer chain as it is close to the conduction band of the chlorine layer ${ }^{8}$. In addition to the STM topography, Fig. 2a shows $\mathrm{d} / \mathrm{d} V$ spectra measured along the trimer chain (see also Supplementary Note 2). The localized states at the domain wall are clearly visible at the bias of around $3.5 \mathrm{~V}$. The localized state can be clearly visualized also in the spatially-
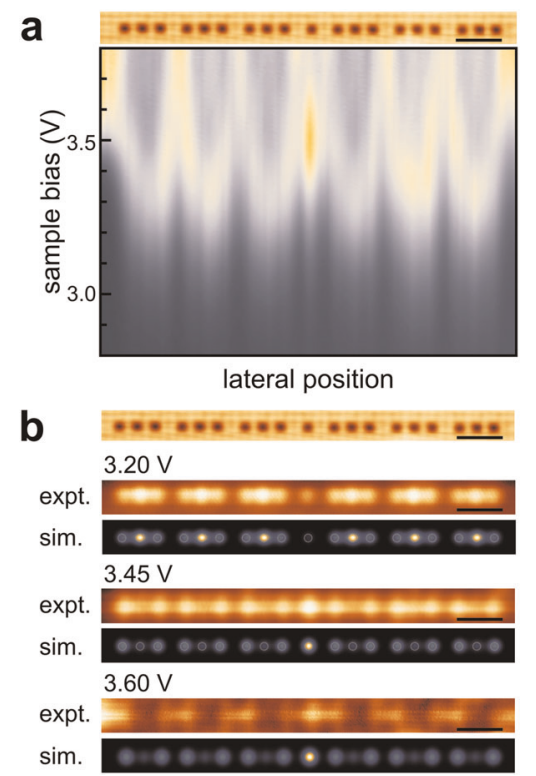

Fig. 2 Experimental realization of a trimer chain. a Stacked contour plot of $d / / d V$ spectra taken along a trimer chain. The chain topography is shown on top for reference (bias $0.5 \mathrm{~V}$, current $1 \mathrm{nA}$ ). b STM topography of trimer (top), experimental $\mathrm{d} / / \mathrm{d} V$ and simulated LDOS maps at the biases indicated in the figure. $\mathrm{d} / / \mathrm{d} V$ maps are recorded in the constant-height mode (determined by set-point current of $1 \mathrm{nA}$ ). Theoretical plots are based on a tight-binding model with $t_{1}=0.14 \mathrm{eV}, t_{2}=t_{3}=0.04 \mathrm{eV}$. Scale bars, $2 \mathrm{~nm}$.

resolved $\mathrm{d} / \mathrm{d} V$ maps (Fig. 2b), where low energy maps show intensity over the whole chain and the domain wall states are visible at the biases corresponding to energies close to the on-site energy (in line with TB predictions). Data for another type of a domain wall in the trimer chain is shown in the Supplementary Note 3.

With atomic-level control, we can move away from the perfect dimerization or trimerization and tune the hoppings between the bulk chains and the domain wall. In the case of a dimer chain, this is expected to have no effect on the energy spectrum as the domain wall state is at zero energy due to symmetry reasons. However, in the case of a trimer chain, we expect that we can move the states within the band gaps. This is illustrated in Fig. 3, where panel a shows the calculated energies of the domain wall state as the hopping $t_{3}$ onto the domain wall site is tuned. The energies are given w.r.t. the on-site energy. The states remain in the gaps as long as the hopping $t_{3}$ is not much smaller than the weaker hopping $t_{2}$ in the chain. By adjusting the nearest neighbor distances, we experimentally tune the hopping $t_{3}$ from $0.04 \mathrm{eV}$ (weakest) to $0.14 \mathrm{eV}$ (strongest) (see Fig. 3b).

Extracting the energies of the domain wall states directly from the experimental $d / / d V$ is difficult due to the energy broadening of the spectra. In addition, the higher lying states coincide with the conduction band of the chlorine layer and hence cannot be reliably detected (see Supplementary Note 4 for details). Instead, we can demonstrate that the experimental results are consistent with the simulations without freely adjustable parameters. The onsite energy, the energy broadening, and the spatial shape of the square of the wavefunction can be extracted from experiments on single vacancies. We get $3.49 \pm 0.01 \mathrm{~V}$ as the on-site energy, the lineshape is a lorentzian with half-width at half-maximum $\Delta=$ $0.18 \pm 0.01 \mathrm{eV}$, and the spatial shape is given by a gaussian with a full-width at half-maximum of $\Gamma=0.71 a$ ( $a$ is the lattice constant of the $\mathrm{c}(2 \times 2)-\mathrm{Cl}$ structure). The values of the hoppings can be extracted from experiments on isolated dimers, which yields the 

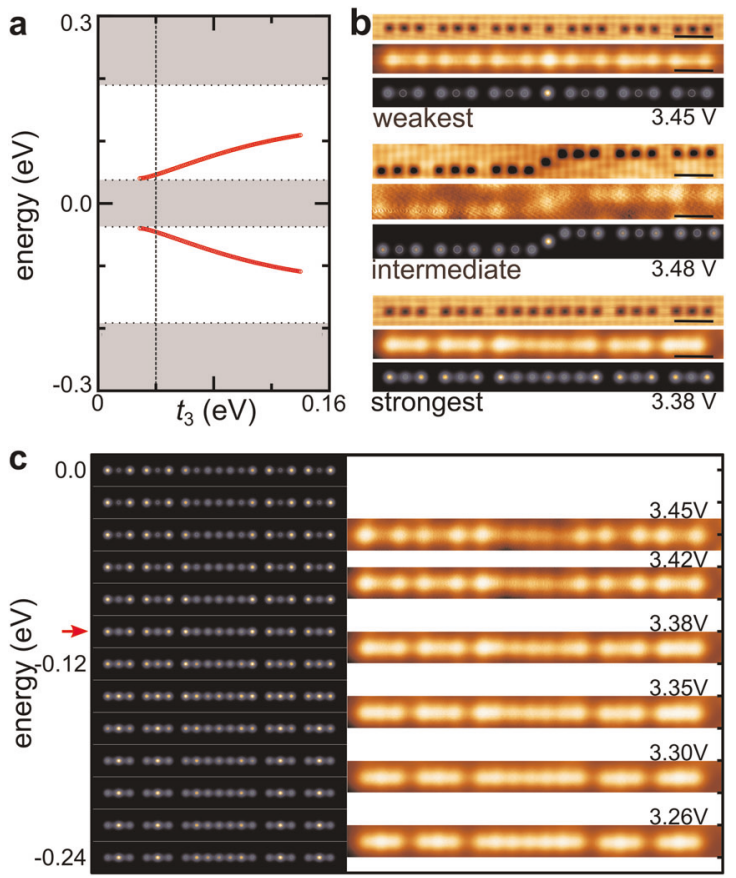

Fig. 3 Tuning the energies of the domain wall states. a Energies (w.r.t. the on-site energy) of the trimer chain in-gap states depending on the hopping $t_{3}$ calculated with a tight-binding model with $t_{1}=0.14 \mathrm{eV}, t_{2}=0.04 \mathrm{eV}$. The shaded areas indicate the three bands of the bulk trimer chain and the dashed vertical line marks $t_{3}=t_{2}$. $\mathbf{b}$ Experimental realizations of modulating the hopping $t_{3}$ into the domain wall site $\left(t_{3}=0.04 ; 0.07 ; 0.14 \mathrm{eV}\right)$. The panels are labeled with the strength of the hopping. In each case, we show STM topography (top, set-point $0.5 \mathrm{~V} / 1 \mathrm{nA}$ ), $\mathrm{d} / / \mathrm{d} V$ map acquired at a bias close to the in-gap state $(3.45,3,48$, and $3.38 \mathrm{~V}$, respectively) and the corresponding simulated LDOS map. Scale bars, $2 \mathrm{~nm}$. c Comparison between the simulated LDOS maps in the case $t_{3}=0.14 \mathrm{eV}$ and the experimental constant-height $\mathrm{d} / / \mathrm{d} V$ maps as a function of energy. The red arrow marks the energy of the in-gap state.

values of $t_{3}=0.04,0.07$, or $0.14 \mathrm{eV}$ for the three structures shown in Fig. 3b.

Using these values, we can simulate a series of LDOS maps and compare them to the experimental ones. This is illustrated in Fig. $3 \mathrm{c}$ and it can be seen that there is an excellent correspondence between the simulations and experimental constant-height $\mathrm{d} / / \mathrm{d} V$ maps (comparison for the weakest coupling is shown in the Supplementary Note 5). In the "bulk" of the chain, the contrast inside the unit cell varies from having most of the intensity on the middle atom to the edge atoms becoming brighter as we approach the on-site energy. On the domain wall segment, we can notice the edge atoms being brightest and the middle of the domain wall being the second brightest feature around the energy corresponding to the domain wall state (marked with the red arrow in Fig. 3c). Similarly for the other domain wall structures, we can match the LDOS maps at the bias corresponding to the domain wall state to the simulated LDOS maps as shown in Fig. 3b. Comparison between isolated monomers and the domain walls segments is shown in the Supplementary Note 6, which further shows that a domain wall in the trimer chain is not equal to an isolated monomer and must be considered to be part of the chain, also in the electronic sense.

The domain walls between trimer chains shifted by one-third (two-thirds) of a unit cell support $e / 3(2 e / 3)$ charge per spin when the lowest band is filled $d^{25,31}$. The possible charge states at a domain wall are integer multiples of $e / 3$, being insensitive to the precise domain wall geometry and in this sense a topological a

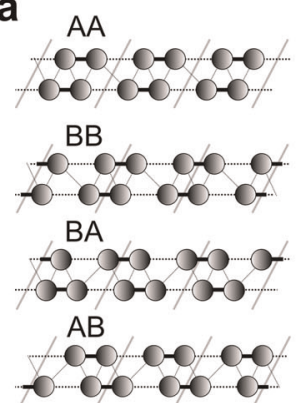

b $\mathrm{AA} \rightarrow \mathrm{BA}$

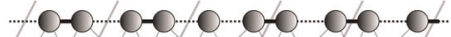

$0-0.0-0-0 \ldots .0-0.0 \%$ domain wall

$A A \rightarrow B B$

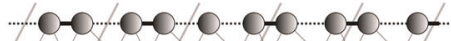

$0-0-0 \% 0,0-0,0$. domain wall $A A \rightarrow A B$

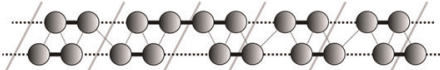
domain wall

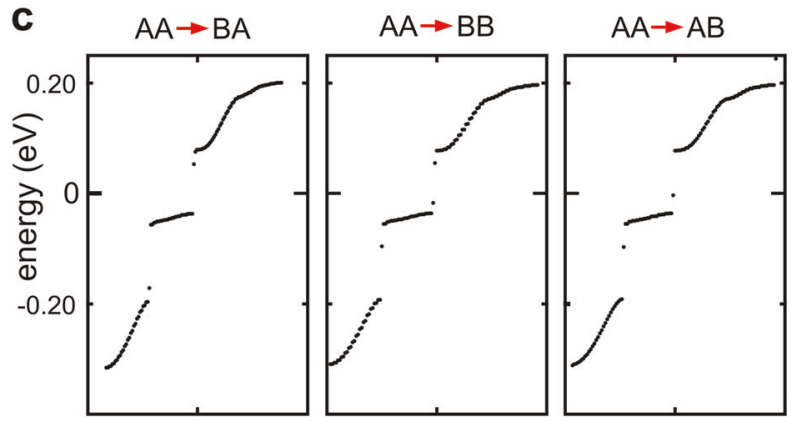

index (eigenstates)

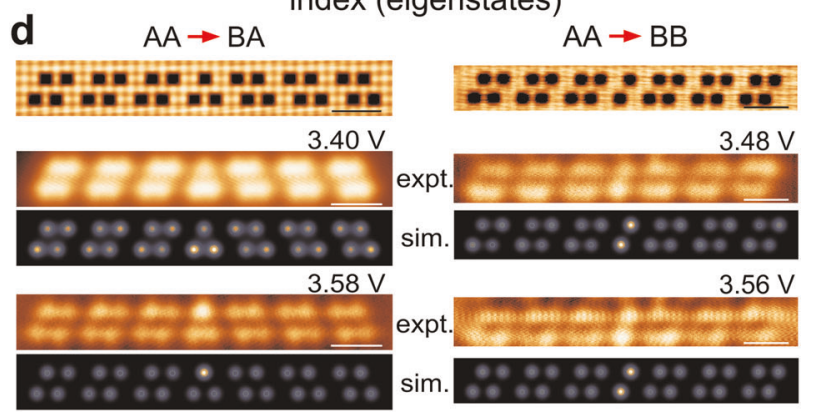

Fig. 4 Domain wall states in coupled dimer chains. a The four ground states of a coupled dimer chains. $\mathbf{b}$ different kinds of domain walls that we have constructed. c The energy diagrams of the domain walls shown in a (calculated with 7 unit cells on each side of the domain wall). d Experimental realizations of the $A A \rightarrow B A$ and $A A \rightarrow B B$ domain walls. Scale bars, $2 \mathrm{~nm}$.

property. Despite the long history of the theoretical studies of trimer chains and their domain wall states, the present work is the first step towards realizing their exotic properties in solid-state devices.

In addition to the tuneable energy of the domain wall states in trimer chains, we consider tuneable domain wall electronic states in coupled dimer chains. This type of systems were demonstrated for the first time in the self-assembled coupled indium dimer chains on silicon ${ }^{21,22,39}$. Atomic-level control allows us to go further and fabricate arbitrary domain walls in coupled dimer chains. Our artificial system readily gives access to domain walls between any of the four different unit cell geometries (Fig. 4a). We have realized all the structures shown in Fig. $4 \mathrm{~b}$ and characterized the domain wall states using $\mathrm{d} / / \mathrm{d} V$ spectroscopy and mapping (see Supplementary Note 7 for additional results). The calculated band structures for the domain walls in Fig. $4 \mathrm{~b}$ are shown in Fig. 4c. The bulk chain has in principle four bands, but using the hoppings corresponding to our experimental system, the two higher energy bands overlap.

Despite that chiral symmetry protecting topological phases and midgap domain wall states in a single dimer chain is broken in coupled chains, the existence of the domain wall states in the coupled chains have a hidden topological origin as pointed out in 
ref. ${ }^{21}$. The different states of the double dimer chain can be expressed in terms of phases $A$ and $B$ (Fig. 1a) to denote the dimerization in each phase, i.e. the coupled dimer chains can be categorized as $A A, A B, B A$, or $B B$, corresponding to both chains being in phase $A$, one being in phase $A$ and the other in phase $B$, etc. The domain wall states can then be classified based on whether they shift the dimerization in only one (e.g., $A A \rightarrow A B$ or $A A \rightarrow B A$ ) or both chains (e.g., $A A \rightarrow B B$ ). As discussed in ref. ${ }^{21}$, the topological origin of the domain wall states is revealed by considering a formal pumping processes between different dimerizations that will transform the system cyclically through configurations $(A A \rightarrow A B \rightarrow B B \rightarrow B A \rightarrow A A)^{21,22}$. The domain wall states can then be interpreted as snap shots of quantum-Hall type chiral edge modes in the formal 2D system of dimerization variable and physical chain. Since the energies of the chiral modes traverse the energy gap depending on the dimerization pattern, their energies are tuneable by the specific geometry of the domain wall. Nevertheless, the domain wall states cannot be removed from the gap by small perturbations. In our case, the coupling between the chains is strong and the spectrum does not closely resemble a decoupled chain. Nevertheless, we can construct analogous domain walls that act differently on the dimerizations of the two chains. Fig. 4d shows examples of domain walls that either shift the dimerization in only a single chain (left) or in both chains (right).

The energies of the domain wall states are not equal in the different cases and they do depend on the values of the hoppings and the domain wall type. To get a general idea on the behavior, we can consider states arising from the domain wall segment and how it couples to the rest of the chain. For example, for the structure $A A \rightarrow B A$, the domain wall itself consists of three lattice sites. There are three states associated with this (see Supplementary Note 8 for a schematic of the energy levels as a function of the interchain coupling $\left(t^{\prime}\right)$ ). For the experimental value of the interchain coupling, the highest energy domain wall state overlaps with a bulk band and hybridizes with it and consequently, the system has two states within the gaps. Furthermore, the energies of these states are influenced on how they are coupled with the bulk chains (similarly to the case of the trimer chain).

In the case of the $A A \rightarrow B B$ domain wall, there are three in-gap states. The two sites forming the domain wall contribute two states that form bonding and antibonding combinations (the lowest and highest in energy, respectively). The third in-gap state is a hybrid between the domain wall and the middle band. We show the calculated wavefunctions of these states in Supplementary Fig. 12. Analogous arguments can be made for the rest of the possible domain wall structures. Again, the experimental results are fully in-line with the TB predictions, indicating that the simple model can be used to design more complicated structures.

In conclusion, we have demonstrated engineering domain wall states in artificial structures fabricated with atomic-level control. Trimer chains allow fabricating domain walls where energy level positions can be tuned through the coupling between the bulk chain and the domain wall site. More complicated structures that can be realized in coupled dimer chains allow domain wall states with additional degrees of freedom (chirality). In the future, extending the atomic manipulation using automated schemes ${ }^{7,9}$ will make it possible to test ideas on using domain wall states with fractional charges (depending on the chemical potential of the system), topological charge pumping and other exotic quantum devices.

\section{METHODS}

\section{Sample preparation}

All sample preparations and experiments were carried out in an ultrahigh vacuum system with a base pressure of $\sim 10^{-10} \mathrm{mbar}$. The (100)-terminated copper single crystal was cleaned by repeated cycles of $\mathrm{Ne}^{+}$sputtering at $1.5 \mathrm{kV}$, annealing to $600^{\circ} \mathrm{C}$. To prepare the chloride structure, anhydrous $\mathrm{CuCl}_{2}$ was deposited from an effusion cell held at $300^{\circ} \mathrm{C}$ onto the warm crystal $\left(T \approx 150-200^{\circ} \mathrm{C}\right)$ for $180 \mathrm{~s}$. The sample was held at the same temperature for $10 \mathrm{~min}$ following the deposition.

\section{STM experiments}

After the preparation, the sample was inserted into the low-temperature STM (Unisoku USM-1300) and all subsequent experiments were performed at $T=4.2 \mathrm{~K}$. STM images were taken in the constant current mode. $\mathrm{d} / / \mathrm{d} V$ spectra were recorded by standard lock-in detection while sweeping the sample bias in an open feedback loop configuration, with a peak-to-peak bias modulation of $20 \mathrm{mV}$ at a frequency of $709 \mathrm{~Hz}$. Line spectra were acquired in constant height; the feedback loop was not closed at any point between the acquisition of the first and last spectra. Manipulation of the chlorine vacancies was carried out as described previously, 7 . The tip was placed above a $\mathrm{Cl}$ atom adjacent to a vacancy site at $0.5 \mathrm{~V}$ bias voltage and the current was increased to $1-2 \mu \mathrm{A}$ with the feedback circuit engaged. The tip was then dragged towards the vacancy site at a speed of up to $250 \mathrm{pm} \mathrm{s}^{-1}$ until a sharp jump in the $z$-position of the tip was observed. This procedure lead to the $\mathrm{Cl}$ atom and the vacancy site exchanging positions with high fidelity.

\section{DATA AVAILABILITY}

All relevant data are available from the authors upon request.

Received: 25 February 2019; Accepted: 20 February 2020; Published online: 12 March 2020

\section{REFERENCES}

1. Eigler, D. M. \& Schweizer, E. K. Positioning single atoms with a scanning tunneling microscope. Nature 344, 524-526 (1990).

2. Crommie, M. F., Lutz, C. P. \& Eigler, D. M. Confinement of electrons to quantum corrals on a metal surface. Science 262, 218-220 (1993).

3. Manoharan, H. C., Lutz, C. P. \& Eigler, D. M. Quantum mirages formed by coherent projection of electronic structure. Nature 403, 512-515 (2000).

4. Gomes, K. K., Mar, W., Ko, W., Guinea, F. \& Manoharan, H. C. Designer Dirac fermions and topological phases in molecular graphene. Nature 483, 306-310 (2012).

5. Celotta, R. J. et al. Invited article: Autonomous assembly of atomically perfect nanostructures using a scanning tunneling microscope. Rev. Sci. Inst. 85, 121301 (2014).

6. Fölsch, S., Martinez-Blanco, J., Yang, J., Kanisawa, K. \& Erwin, S. C. Quantum dots with single-atom precision. Nat. Nanotechnol. 9, 505-508 (2014).

7. Kalff, F. E. et al. A kilobyte rewritable atomic memory. Nat. Nanotechnol. 11, 926-929 (2016).

8. Drost, R., Ojanen, T., Harju, A. \& Liljeroth, P. Topological states in engineered atomic lattices. Nat. Phys. 13, 668-671 (2017)

9. Girovsky, J. et al. Emergence of quasiparticle Bloch states in artificial crystals crafted atom-by-atom. SciPost Phys. 2, 020 (2017).

10. Slot, M. R. et al. Experimental realization and characterization of an electronic Lieb lattice. Nat. Phys. 13, 672-676 (2017).

11. Fölsch, S., Hyldgaard, P., Koch, R. \& Ploog, K. H. Quantum confinement in monatomic Cu chains on Cu(111). Phys. Rev. Lett. 92, 056803 (2004).

12. Repp, J., Meyer, G., Paavilainen, S., Olsson, F. E. \& Persson, M. Scanning tunneling spectroscopy of $\mathrm{Cl}$ vacancies in $\mathrm{NaCl}$ films: strong electron-phonon coupling in double-barrier tunneling junctions. Phys. Rev. Lett. 95, 225503 (2005).

13. Schuler, B. et al. Effect of electron-phonon interaction on the formation of onedimensional electronic states in coupled $\mathrm{Cl}$ vacancies. Phys. Rev. B 91, 235443 (2015).

14. Paavilainen, S., Ropo, M., Nieminen, J., Akola, J. \& Räsänen, E. Coexisting honeycomb and kagome characteristics in the electronic band structure of molecular graphene. Nano Lett. 16, 3519-3523 (2016).

15. Kempkes, S. N. et al. Design and characterization of electrons in a fractal geometry. Nat. Phys. 15, 127-131 (2019).

16. Hasan, M. Z. \& Kane, C. L. Colloquium: topological insulators. Rev. Mod. Phys. 82, 3045-3067 (2010)

17. Mourik, V. et al. Signatures of Majorana fermions in hybrid superconductorsemiconductor nanowire devices. Science 336, 1003-1007 (2012). 
18. Nadj-Perge, S. et al. Observation of Majorana fermions in ferromagnetic atomic chains on a superconductor. Science 346, 602-607 (2014).

19. Ruby, M. et al. End states and subgap structure in proximity-coupled chains of magnetic adatoms. Phys. Rev. Lett. 115, 197204 (2015)

20. Kim, H. et al. Toward tailoring Majorana bound states in artificially constructed magnetic atom chains on elemental superconductors. Sci. Adv. 4, eaar5251 (2018).

21. Cheon, S., Kim, T.-H., Lee, S.-H. \& Yeom, H. W. Chiral solitons in a coupled double Peierls chain. Science 350, 182-185 (2015).

22. Kim, T.-H., Cheon, S. \& Yeom, H. W. Switching chiral solitons for algebraic operation of topological quaternary digits. Nat. Phys. 13, 444-447 (2017).

23. Cao, T., Zhao, F. \& Louie, S. G. Topological phases in graphene nanoribbons: junction states, spin centers, and quantum spin chains. Phys. Rev. Lett. 119 076401 (2017).

24. Sato, M. \& Ando, Y. Topological superconductors: a review. Rep. Prog. Phys. 80 076501 (2017).

25. Heeger, A. J., Kivelson, S., Schrieffer, J. R. \& Su, W. P. Solitons in conducting polymers. Rev. Mod. Phys. 60, 781-850 (1988).

26. Jackiw, R. \& Rebbi, C. Solitons with fermion number $1 / 2$. Phys. Rev. D 13, 3398-3409 (1976).

27. Kane, C. in Contemporary Concepts of Condensed Matter Science (eds Franz, M. \& Molenkamp, L.) Vol. 6, 3-34 (Elsevier, 2013).

28. Meier, E. J., An, F. A. \& Gadway, B. Observation of the topological soliton state in the Su-Schrieffer-Heeger model. Nat. Commun. 7, 13986 (2016).

29. Gröning, O. et al. Engineering of robust topological quantum phases in graphene nanoribbons. Nature 560, 209-213 (2018).

30. Rizzo, D. J. et al. Topological band engineering of graphene nanoribbons. Nature 560, 204-208 (2018).

31. Su, W. P. \& Schrieffer, J. R. Fractionally charged excitations in charge-density-wave systems with commensurability 3. Phys. Rev. Lett. 46, 738-741 (1981).

32. Rice, M. J. \& Mele, E. J. Elementary excitations of a linearly conjugated diatomic polymer. Phys. Rev. Lett. 49, 1455-1459 (1982).

33. Jackiw, R. \& Semenoff, G. Continuum quantum field theory for a linearly conjugated diatomic polymer with fermion fractionization. Phys. Rev. Lett. 50, 439-442 (1983)

34. Kivelson, S. Solitons with adjustable charge in a commensurate Peierls insulator. Phys. Rev. B 28, 2653-2658 (1983).

35. Martinez Alvarez, V. M. \& Coutinho-Filho, M. D. Edge states in trimer lattices. Phys. Rev. A 99, 013833 (2019).

36. Kim, T.-H. \& Yeom, H. W. Topological solitons versus nonsolitonic phase defects in a quasi-one-dimensional charge-density wave. Phys. Rev. Lett. 109, 246802 (2012).

37. Shim, H., Lee, G., Hyun, J.-M. \& Kim, H. Cooperative interplay between impurities and charge density wave in the phase transition of atomic wires. New J. Phys. 17, 093026 (2015).

38. Lee, S., Woo, J. \& Lee, G. Influence of $\mathrm{Pb}$ on an $\ln / \mathrm{Si}(111)$ surface on the phase transition and the surface structure. J. Kor. Phys. Soc. 70, 740-744 (2017).

39. Lee, G., Shim, H., Hyun, J.-M. \& Kim, H. Intertwined solitons and impurities in a quasi-one-dimensional charge-density-wave system: In/Si(111). Phys. Rev. Lett 122, 016102 (2019)

40. Thouless, D. J. Quantization of particle transport. Phys. Rev. B 27, 6083-6087 (1983)

41. Wang, L., Troyer, M. \& Dai, X. Topological charge pumping in a one-dimensional optical lattice. Phys. Rev. Lett. 111, 026802 (2013).
42. Guo, H. Dimerization, trimerization and quantum pumping. Phys. Lett. A 378, 1316-1320 (2014).

\section{ACKNOWLEDGEMENTS}

This research made use of the Aalto Nanomicroscopy Center (Aalto NMC) facilities and was supported by the European Research Council (ERC-2017-AdG no. 788185 "Artificial Designer Materials"), Academy of Finland (Academy professor funding no. 318995 and 320555, and Academy postdoctoral researcher no. 309975), and the Aalto University Centre for Quantum Engineering (Aalto CQE).

\section{AUTHOR CONTRIBUTIONS}

M.H., S.K., and P.L. conceived, planned, and carried out the experiments. M.H. analyzed the experimental data. T.O. and R.D. contributed to the theoretical analysis of the systems. All authors jointly authored, commented, and corrected the paper.

\section{COMPETING INTERESTS}

The authors declare no competing interests.

\section{ADDITIONAL INFORMATION}

Supplementary information is available for this paper at https://doi.org/10.1038/ s41535-020-0219-3.

Correspondence and requests for materials should be addressed to P.L.

Reprints and permission information is available at http://www.nature.com/ reprints

Publisher's note Springer Nature remains neutral with regard to jurisdictional claims in published maps and institutional affiliations.

\section{(i)}

Open Access This article is licensed under a Creative Commons Attribution 4.0 International License, which permits use, sharing, adaptation, distribution and reproduction in any medium or format, as long as you give appropriate credit to the original author(s) and the source, provide a link to the Creative Commons license, and indicate if changes were made. The images or other third party material in this article are included in the article's Creative Commons license, unless indicated otherwise in a credit line to the material. If material is not included in the article's Creative Commons license and your intended use is not permitted by statutory regulation or exceeds the permitted use, you will need to obtain permission directly from the copyright holder. To view a copy of this license, visit http://creativecommons. org/licenses/by/4.0/.

(c) The Author(s) 2020 